\title{
Impact of COVID-19 pandemic on the psychological status of infertile patients who had in vitro fertilization treatment interrupted or postponed: a cross-sectional study
}

\author{
Fabio Barra ${ }^{1}$, Valentina Lucia La Rosa ${ }^{2}$, Salvatore Giovanni Vitale ${ }^{3}$, Elena Commodari ${ }^{3}$, \\ Michele Altieri ${ }^{4}$, Carolina Scala ${ }^{5}$, and Simone Ferrero ${ }^{6}$ \\ ${ }^{1}$ University of Genoa \\ ${ }^{2}$ Università degli Studi di Catania \\ ${ }^{3}$ University of Catania \\ ${ }^{4}$ Ospedale Policlinico San Martino Istituto di Ricovero e Cura a Carattere Scientifico per \\ l'Oncologia \\ ${ }^{5}$ Istituto Giannina Gaslini Istituto Pediatrico di Ricovero e Cura a Carattere Scientifico \\ ${ }^{6}$ Affiliation not available
}

August 22, 2020

\begin{abstract}
Objective: To investigate the changes in the psychological status of infertile women and men who had infertility treatment interrupted or postponed because of the COVID-19 pandemic. Design: Cross-sectional study (NCT04400942) performed between April and June 2020. Population: Patients having in-vitro fertilization (IVF) treatments at our institution interrupted or postponed due to the COVID-19 emergency. Methods: An electronic survey was e-mailed to investigate presence, severity of anxiety and depression using the Generalized Anxiety Disorder-7 (GAD-7) and the Patient Health Questionnaire-9 (PHQ-9). Subjects with pre-existing psychiatric disorders diagnosed by DSM-V criteria were excluded from the analysis. Main Outcome Measures: Presence, severity of anxiety and depression; risk factors related to these psychological disorders. Results: Overall, 524 out of 646 patients $(80.9 \%)$ completed the survey. The prevalence of anxiety and/or depression was significantly higher in women $(41.5 \%, \mathrm{p}=0.012)$, in particular, if aged more than 35 years $(50.2 \%, \mathrm{p}=0.023)$ and with a previous IVF attempt $(57.6 \%$, $\mathrm{p}=0.031$ ). The occurrence of these psychological disorders was significantly associated with the time spent on COVID-19 related news per day ( $>1$ hour per day, $\mathrm{p}=0.034)$ and partner with evidence of psychological disorder $(\mathrm{p}=0.017)$ and, in females, with a diagnosis of poor ovarian reserve $(\mathrm{p}=0.052)$, diagnosis of endometriosis $(\mathrm{p}<0.001)$ or uterine fibroids $(\mathrm{p}=0.037)$. Conclusions: The psychological impact of the COVID-19 pandemic on the infertile couples who should have undergone IVF treatment was significant. IVF centers should systematically offer these couples an adequate psychological counseling to improve quality of life and mental health. Funding: - Keywords: INFERTILITY: ASSISTED CONCEPTION; PSYCHOLOGY
\end{abstract}

\section{Tweetable abstract}

The psychological impact of COVID-19 on infertile couples who had IVF treatment interrupted or postponed is significant.

\section{Introduction}

The remarkable increase in the number of infections by severe acute respiratory syndrome coronavirus 2 (SARS-CoV-2) worldwide raised the prospect of massive hospitalizations that few healthcare systems would face. During the COVID-19 pandemic, the urgent need to avoid a collapse in the healthcare system has been 
the justification for the implemented measures, and reproductive medicine societies, as well as regulatory authorities, decisively followed by issuing guidance based on expert best judgment (1). The recommendations for practitioners include suspension of initiation of assisted reproductive technology (ART) treatments, suspension of non-urgent diagnostic procedures, and elective surgical operations.

In the earliest stages of the pandemic, the European Society of Human Reproduction and Embryology (ESHRE) and the American Society for Reproductive Medicine (ASRM) suggested discontinuing new fertility procedures, such as ovulation induction, intrauterine insemination (IUI), and in vitro fertilization (IVF), as well as non-urgent gamete cryopreservation, cancellation of all fresh or frozen embryo transfers. Exceptions were those patients who are currently 'in-cycle' or who require urgent fertility preservation due to cancer treatment (2). More recently, in Europe, with successful mitigation strategies in some areas and the emergence of additional data, societies have allowed gradual resumption of ART procedures (3).

Infertility is already a stressful experience. Indeed, it has been widely documented that the experience of infertility has a significant impact on the psychological wellbeing of both partners and problems such as low self-esteem, sexual distress, depression, guilt, anxiety, frustration, and relational problems within the couple frequently occur in infertile couples $(4,5)$. Furthermore, psychological factors play an important role in determining the success of assisted reproduction treatments, and the failure of these techniques can further impair the mental health of individuals and couples $(6,7)$. In addition to this stressful condition, patients who have decided to proceed with an IVF in the last couple of months also faced the psychological impact of the COVID-19 pandemic, which has drastically impacted everyone's daily lives because of quarantine, social distancing, restrictions in traveling, treatment cancellation along with constant fear for their own and their families in danger (8). This feeling of stress, anxiety, and depression is likely to impact the emotional well-being of couples who should have undergone IVF treatment during the COVID-19 pandemic.

In light of these considerations, this cross-sectional study aimed to explore the changes in the psychological status of infertile patients whose IVF treatments have been interrupted or postponed due to the COVID-19 emergency. In particular, we hypothesized that the COVID-19 experience led to a significant increase in the anxiety and depression levels of these couples and especially of women, who are more subject to the psychological consequences of infertility and its treatments.

\section{Materials and Methods}

This cross-sectional study enrolled all the patients in our institution (Piazza Della Vittoria 14 Srl; Genoa; Italy) whose IVF treatments have been interrupted or postponed due to the COVID-19 pandemic. Patients were identified, and demographic data were prospectively collected using dedicated software (Gineko, Cosa Srl, Rome, Italy) between 1 April 2020 and 10 June 2020.

The local Ethical Committee approved the study protocol (402/2020 CER Liguria), and all research was performed following relevant guidelines and regulations. All the women gave their informed consent for the collection and use of their data for research purposes. The study was registered in ClinicalTrials.gov (NCT04400942).

An anonymous electronic survey on Google Forms web application (Mountain View, California, United States) was e-mailed to the eligible study patients. A reminder e-mail was sent every week until the end of the study period. The purpose of this survey was explained to all participants with a brief introduction. Participants were asked to sign a privacy policy consent at the beginning. Survey participation was voluntary, and no incentives were offered.

The survey was composed of two distinct sections: the first part assessed presence and severity of anxiety and depression using the Generalized Anxiety Disorder-7 (GAD-7) and the Patient Health Questionnaire9 (PHQ-9); the second part evaluated the perspective of patients about restarting IVF treatment, even changing referral ART center. Subjects with pre-existing psychiatric disorders diagnosed by using DSM-V were excluded from the sample (9).

The GAD-7 represents a validated item based on seven items referring to DSM-IV criteria for assessing 
anxiety. The whole scale score can range from 0 to 21 , and cut-off scores for mild, moderate, and severe anxiety symptoms are 5, 10, and 15, respectively (10). The PHQ-9 is a validated item based on nine items referring to DSM-IV criteria for assessing depression. The whole scale score can range from 0 to 27 , and cut-off scores for mild, moderate, moderately severe, severe depression symptoms are 5, 10, 15, and 20, respectively (11). For both questionnaires, response options are "not at all", "several days", "more than half the days", and "nearly every day", scored as $0,1,2$, and 3, respectively.

After the closing date for questionnaire submissions, results were downloaded as a CSV (comma-separated values) file to be categorized via Excel (version 16.39; Microsoft Corporation, Redmond, USA). Statistical analysis was carried out using Statistics Package for Social Sciences (SPSS, version 24.0 Chicago, IL, USA). Results of the survey were reported according to the CHERRIES Guidelines (12).

\section{Data analysis}

Continuous data were verified for normality using the Kolmogorov-Smirnov test. According to the distribution of the variables, descriptive statistics were reported as mean \pm standard deviation or median and interquartile range (IRQ). Unpaired or paired t-test was employed for analyzing continuous data and the chi-squared test for categorical data. The Mann-Whitney U test was employed to compare the differences between two independent groups in case of dependent variables, which were not normally distributed. Logistic regression analysis was used to examine the odds ratio (OR) with a 95\% CI of (categorical or continuous) independent variables with one dichotomous dependent variable. Correlation measurements were tested using Spearman's Rho test. A two-sided P-value [?] 0.05 was considered statistically significant.

\section{Results}

Overall, 524 (308 women; 216 men) out of 646 patients completed the questionnaire (response rate: 81.1\%; $95 \%$ C.I., $77.7 \%-84.1 \%$; Figure 1). The mean (+- SD) age of the women and men included in the study was $37.3(+-4.6)$ and 38.4 (+- 6.2) years, respectively. Overall, 187 couples were included in the study analysis, with a median number of previous failed IVF cycles of 2 (range, 0-8). Fifty-six couples (29.9\%) were undergoing heterologous fertilization. The other demographic characteristics of the study population are reported in Table 1.

The global prevalence of anxiety or depression or both was $38.9 \%$ (204 of 524; $95 \%$ C.I., $34.7 \%-43.3 \%$ ). The prevalence of anxiety or depression or both was significantly higher in women than in men $(45.5 \%$ vs. $31.0 \%$; $\mathrm{p}=0.001)$, in women aged more than 35 years $(49.3 \%$ vs. $46.3 \%$; $=0.036)$ and with a previous IVF attempt $(57.7 \%$ vs. $24.1 \% ; \mathrm{p}<0.001)$.

Anxiety was detected in 114 patients (21.8\%; 95\% C.I., 18.3\%-25.5\%) with a median total score on GAD-7 of 11 (interquartile range, IQR: 8-15; Figure 2). Among these patients, the most frequent symptom severity was mild ( $\mathrm{n}=58 ; 11.1 \% ; 95 \%$ C.I., $8.5 \%-14.1 \%)$. A higher proportion of women suffered from moderate anxiety ( $8.8 \%$ vs. $1.3 \% ; \mathrm{p}<0.001$; Table 2 ). Depression was detected in 93 patients (17.7\%; $95 \%$ C.I., $14.8 \%$ - $21.3 \%$ ) with a median total score on PHQ-9 of 10 (IQR: 9-21; Figure 2). Among these patients, the most frequent symptom severity was moderate $(\mathrm{n}=63 ; 12.0 \% ; 95 \%$ C.I., $9.2 \%-14.8 \%$; Table 2$)$. The proportion of women and men affected by mild and moderate depression was not significantly different $(\mathrm{p}=0.707$ and $\mathrm{p}=0.397$, respectively), whereas a higher proportion of men suffered from moderately severe depression $(\mathrm{P}=0.007)$. Considering all the patients, there was a significant weak direct correlation between scores obtained at GAD-7 and those obtained at PHQ-9 $(\mathrm{R}=0.214 ; \mathrm{p}<0.001)$. Only 38 out of 524 patients (7.3\%; 95\% C.I., 5.2\%-9.8\%) had a concomitant diagnosis of depression and anxiety.

In both females and males, the occurrence of anxiety and/or depression was significantly associated with time spent on COVID-19 related news per day ( $>1$ hour per day, $\mathrm{p}=0.034)$ and partner with evidence of psychological disorder $(\mathrm{p}=0.017$; Table 3$)$. The level of instruction and a previous parity did not significantly correlate with the occurrence of anxiety and/or depression ( $\mathrm{p}=0.383$ and $\mathrm{p}=0.679$, respectively). Specifically for females, the occurrence of anxiety and/or depression was significantly associated with poor ovarian reserve $(\mathrm{p}=0.032)$, diagnosis of endometriosis $(\mathrm{p}<0.001)$, diagnosis of uterine fibroids $(\mathrm{p}=0.040$; Table 3$)$. A previous 
failed IVF cycle was not associated with the occurrence of these psychological disorders ( $\mathrm{p}=0.910)$; similarly, there was no direct correlation with the number of previous failed IVF cycles $(\mathrm{R}=0.049 ; \mathrm{p}=0.388)$.

Overall, 196 patients (37.4\%; 95\% C.I., 33.4\%-41.6\%) declared that they would like to undergo the IVF treatment despite the COVID-19 pandemic. Three hundred ninety-one patients $(74.6 \%$; 95\% C.I., $70.2 \%$ $78.2 \%$ ) reported that they would be reassured if they knew when they could restart the IVF treatment; 206 patients (39.3\%; 95\% C.I., 35.2\%-43.6\%) declared that they would change the IVF center if they could restart the IVF treatment immediately.

\section{Discussion}

Discontinuation of IVF cycles has been part of the radical transformation of healthcare provision to enable the reallocation of staff and resources to deal with the COVID-19 pandemic. At the best of our knowledge, this is the first study investigating the changes in the psychological status of infertile patients whose IVF treatments have been interrupted or postponed due to the COVID-19 pandemic. This survey revealed that the COVID-19 epidemic caused a sharp increase in the prevalence of anxiety and depression among infertile patients undergoing IVF. More specifically, our analysis demonstrated that more than one-third of patients referring to our IVF center had evidence of anxiety or depression. Furthermore, a higher proportion of patients were anxious than depressed (21.8\% vs. $17.7 \%)$, although the mean anxiety severity was lower than that of depression (mild severity score: $11.1 \%$ vs. $5.0 \%$; moderate severity score: $10.1 \%$ vs. $12.0 \%$ ); however, only about $1 \%$ of patients with evidence of these phycological disorders showed severe symptoms (Table 2).

These results are in line with the existing literature on the topic according to which depression and anxiety are frequently associated with infertility, and they may worsen during assisted reproduction treatments (6). In this regard, in a recent study on women undergoing infertility treatments, $65.9 \%$ scored in the clinical range for depression and $75.9 \%$ for anxiety; these psychological disorders were higher for women who did not have a successful ART procedure (13). Moreover, approximately $13 \%$ of infertile women reported taking antidepressant medications (14). Haimovici et al. found psychopathological symptoms in $72 \%$ of the couples and more commonly in females. Female and male stress was associated with stress, anxiety, and depression in the respective partner. Notably, a lower concentration of serum transforming growth factor-beta (TGF- $\beta$ ) and a higher level of cervicovaginal interleukin (IL) -6 and IL-1 $\beta$ correlated with the presence of stress (15). Interestingly, Gourounti et al. underlined that low perception of personal control and avoidant coping style may be positively associated with fertility-related stress and state anxiety. In contrast, a problem-appraisal coping style may be negatively related to fertility-related stress and depressive symptomatology scores (16).

COVID-19 disease is an unprecedented global situation that is drastically changing everyone's daily life and perspective. The stress caused by the COVID-19 pandemic is unprecedented in modern history. Indeed, it was an unexpected event whose consequences continue to threaten everyone's present life and future, including the loss of normal life due to the lockdown, financial insecurity, and social isolation. In this regard, recent studies on this topic confirmed the severe psychological repercussions of the emergency linked to the spread of COVID-19 in many sections of the population such as healthcare professionals and students $(8$, 17-19).

In this scenario, it is reasonable to hypothesize that the feelings of stress, anxiety, and depression related to the COVID-19 pandemic can have had a significant impact on the emotional well-being of couples who should have undergone IVF treatment during the emergency. In this regard, a short communication published during the COVID-19 pandemic presented data about an anonymous cross-sectional online survey sent to 10,481 patients who attended a large university-affiliated infertility practice in the USA. At three different timepoints, respondents indicated infertility as the most frequent top stressor, causing anxiety and depression. COVID-19 was the third most common stressor among the respondents in the early stages of the pandemic (first days of March); nevertheless, in the latest period (first days of June), COVID-19 caused stress with an incidence similar to infertility ( $63 \%$ and $66 \%$, respectively). In this study, only $6 \%$ of patients stated that infertility treatment, including IVF, should not be offered during the COVID-19 pandemic. In agreement with this data, our study showed that the occurrence of anxiety and/or depression was significantly associated 
with time spent on COVID-19 related news per day.

The impact of the IVF interruption due to the COVID-19 pandemic has been evaluated on 271,438 ovarian stimulation cycles in the UK by estimating the effect of age as a continuous, yet non-linear, function on the cumulative live birth rate. This model was recalibrated to cumulative live birth rates reported for the 135,673 stimulation cycles undertaken in the USA in 2016, with live birth follow-up to October 2018. The authors reported that discontinuation of fertility treatment for even one month in the USA could result in 369 fewer women having live birth, due to the increase in patients' age during the shutdown (20). On the opposite, another study preliminary reported that in women with diminished ovarian reserve (AMH $<1.1$ $\mathrm{ng} / \mathrm{ml}$ ) there was no difference in the live birth rate among women who initiated their IVF cycle within 90 days of their first visit due to COVID-19 pandemic compared to women who did procedures 91-180 days after initial consultation (21). Although the psychological impact of ART delayed was not evaluated in the study, these latter results may be reassuring to women with poor ovarian reserve, who may feel particularly anxious and depressed to begin their treatment and become frustrated when unexpected delays occur, as demonstrated by the logistic regression analysis of our data.

In the current study, women reported a higher rate of anxiety and/or depression when compared with men (41.5 vs. $30.6 \%)$. It has been well documented that infertility is commonly linked with depression, particularly in women. Infertility diagnosis and the subsequent stress of treatments have been linked with increased infertility distress (22). Moreover, a large Spanish study population explored the psychological impact of the COVID-19 pandemic in the general adult population; women demonstrated to have a significant psychological impact, with not a negligible rate of stress, anxiety, and depression (23). However, men undergoing fertility treatment may also experience anxiety and stress (24). To this purpose, in our study, $30.6 \%$ of men experienced anxiety and/or depression. During COVID-19 lockdown, the health and psychological consequences of not offering andrological services should be considered. Indeed, the lockdown of andrological services may have a devastating psychological impact on men undergoing ART treatment.

Psychological interventions may have a critical role in lowering psychological distress in patients referring to IVF centers (25), in particular, during the COVID-19 pandemic. A cognitive-behavioral may be the most efficient way to achieve both goals. Even more so, during the COVID-19 epidemic, IVF center must strengthen the psychological counseling for couples to improve their quality of life and mental health. To this purpose, specific strategies may support multiple times across the treatment trajectory by various methods (i.e., website, handouts, personal referral) and by multiple providers (i.e., psychologists, medical assistants, nurses, physicians).

Our study has some important strengths. As already underlined, it is one of the first studies investigating the psychological impact of the COVID-19 emergency on the infertile patients whose IVF treatments have been interrupted or postponed. Furthermore, our sample is relatively large, and consequently, our results can be considered significant.

However, there are also some limitations. First, this is a cross-sectional study, and we did not analyze the prevalence of anxiety and/or depression in patients undergoing IVF at our institution before the COVID-19 pandemic. Therefore, we cannot establish an exact causal relationship between the investigated variables. Secondly, we used an internet-based questionnaire with self-reported measures, so it was not possible to exclude a potential influence of self-report bias on our results. Finally, despite a large number of respondents, it is possible that this is not a general representative sample, as the survey was distributed in our local population; thus, the responses may not be generalizable in all the Italian regions or different countries. In the next months, a longitudinal follow-up would help track the changes in anxiety and depression levels at various stages of the epidemic.

\section{Conclusions}

In conclusion, according to the results of this study, the psychological impact of the COVID-19 pandemic on the infertile couples whose IVF treatments were postponed or interrupted because of the emergency was significant. For this reason, it is essential that IVF centers systematically offer these couples an adequate psy- 
chological counseling to improve their quality of life and mental health as well as to reduce the psychological impact of the COVID-19 pandemic as much as possible.

\section{Acknowledgements}

None

\section{Conflict of Interest}

The authors have no conflicts of interest to declare.

\section{Authors' Contributions}

F.B. designed the study, collected data, and drafted the article; V.L.L.R. performed statistical analyses; E.C. and C.S. critically revised the manuscript; S.G.V. and M.A. contributed to the data analysis and drafted the article; S.F. supervised the study. All authors read and approved the final manuscript.

\section{Details of Ethics Approval}

The local Ethical Committee approved the study protocol (402/2020 CER Liguria), and all research was performed following relevant guidelines and regulations. All the women gave their informed consent for the collection and use of their data for research purposes. The study was registered in ClinicalTrials.gov (NCT04400942).

\section{Funding}

No funding was obtained for this study.

\section{References}

1. Armocida B, Formenti B, Ussai S, Palestra F, Missoni E. The Italian health system and the COVID-19 challenge. Lancet Public Health. 2020;5(5):e253.

2. Veiga A, Gianaroli L, Ory S, Horton M, Feinberg E, Penzias A. Assisted reproduction and COVID-19: A joint statement of ASRM, ESHRE and IFFS. Fertil Steril. 2020.

3. Andrabi SW, Jaffar M, Arora PR. COVID-19: New adaptation for IVF laboratory protocols. JBRA Assist Reprod. 2020;24(3):358-61.

4. Vitale SG, La Rosa VL, Rapisarda AMC, Laganà AS. Endometriosis and Infertility: The Impact on Quality of Life and Mental Health. Journal of Endometriosis and Pelvic Pain Disorders. 2017;9(2):112-5.

5. La Rosa VL, Barra F, Chiofalo B, Platania A, Di Guardo F, Conway F, et al. An overview on the relationship between endometriosis and infertility: the impact on sexuality and psychological well-being. J Psychosom Obstet Gynaecol. 2019:1-5.

6. Rooney KL, Domar AD. The relationship between stress and infertility. Dialogues Clin Neurosci. 2018;20(1):41-7.

7. Vitale SG, La Rosa VL, Rapisarda AM, Lagana AS. Psychology of infertility and assisted reproductive treatment: the Italian situation. J Psychosom Obstet Gynaecol. 2017;38(1):1-3.

8. Torales J, O'Higgins M, Castaldelli-Maia JM, Ventriglio A. The outbreak of COVID-19 coronavirus and its impact on global mental health. Int J Soc Psychiatry. 2020;66(4):317-20.

9. American Psychiatric Association. Diagnostic and statistical manual of mental disorders (5th ed.): Arlington; 2013.

10. Jordan P, Shedden-Mora MC, Lowe B. Psychometric analysis of the Generalized Anxiety Disorder scale (GAD-7) in primary care using modern item response theory. PloS one. 2017;12(8):e0182162. 
11. Maroufizadeh S, Omani-Samani R, Almasi-Hashiani A, Amini P, Sepidarkish M. The reliability and validity of the Patient Health Questionnaire-9 (PHQ-9) and PHQ-2 in patients with infertility. Reprod Health. 2019;16(1):137.

12. Eysenbach G. Improving the quality of Web surveys: the Checklist for Reporting Results of Internet E-Surveys (CHERRIES). J Med Internet Res. 2004;6(3):e34.

13. Pasch LA, Holley SR, Bleil ME, Shehab D, Katz PP, Adler NE. Addressing the needs of fertility treatment patients and their partners: are they informed of and do they receive mental health services? Fertil Steril. 2016;106(1):209-15 e2.

14. Pedro J, Vassard D, Malling GMH, Hougaard CO, Schmidt L, Martins MV. Infertility-related stress and the risk of antidepressants prescription in women: a 10-year register study. Hum Reprod. 2019;34(8):1505-13.

15. Haimovici F, Anderson JL, Bates GW, Racowsky C, Ginsburg ES, Simovici D, et al. Stress, anxiety, and depression of both partners in infertile couples are associated with cytokine levels and adverse IVF outcome. Am J Reprod Immunol. 2018;79(4):e12832.

16. Gourounti K, Anagnostopoulos F, Potamianos G, Lykeridou K, Schmidt L, Vaslamatzis G. Perception of control, coping and psychological stress of infertile women undergoing IVF. Reprod Biomed Online. 2012;24(6):670-9.

17. Ramaci T, Barattucci M, Ledda C, Rapisarda V. Social Stigma during COVID-19 and its Impact on HCWs Outcomes. Sustainability. 2020;12(9).

18. Montemurro N. The emotional impact of COVID-19: From medical staff to common people. Brain, behavior, and immunity. 2020;87:23-4.

19. Sandhu P, de Wolf M. The impact of COVID-19 on the undergraduate medical curriculum. Medical education online. 2020;25(1):1764740.

20. Smith ADAC, Gromski PS, Rashid KA, Tilling K, Lawlor DA, Nelson SM. Population implications of cessation of IVF during the COVID-19 pandemic. Reproductive BioMedicine Online. 2020.

21. Romanski PA, Bortoletto P, Rosenwaks Z, Schattman GL. Delay in IVF treatment up to 180 days does not affect pregnancy outcomes in women with diminished ovarian reserve. Hum Reprod. 2020;35(7):1630-6.

22. Peterson BD, Sejbaek CS, Pirritano M, Schmidt L. Are severe depressive symptoms associated with infertility-related distress in individuals and their partners? Hum Reprod. 2014;29(1):76-82.

23. Rodriguez-Rey R, Garrido-Hernansaiz H, Collado S. Psychological Impact and Associated Factors During the Initial Stage of the Coronavirus (COVID-19) Pandemic Among the General Population in Spain. Front Psychol. 2020;11:1540.

24. Esteves SC, Lombardo F, Garrido N, Alvarez J, Zini A, Colpi GM, et al. SARS-CoV-2 pandemic and repercussions for male infertility patients: A proposal for the individualized provision of andrological services. Andrology. 2020.

25. Peterson B, Boivin J, Norre J, Smith C, Thorn P, Wischmann T. An introduction to infertility counseling: a guide for mental health and medical professionals. J Assist Reprod Genet. 2012;29(3):243-8.

Table 1. Demographic characteristics of the study population

\begin{tabular}{lll}
\hline Females $(\mathrm{n}=308)$ & Females $(\mathrm{n}=308)$ & Males $(\mathrm{n}=216)$ \\
\hline Age, mean $( \pm \mathrm{SD})$ & $37.3( \pm 4.6)$ & $38.4( \pm 6.2)$ \\
BMI, mean $( \pm \mathrm{SD})$ & $24.5( \pm 3.1)$ & $24.2( \pm 3.3)$ \\
Smoking, $\mathrm{n}(\%)$ & $56(18.2)$ & $37(17.2)$ \\
Ethnicity, n $(\%)$ & &
\end{tabular}




\begin{tabular}{lll}
\hline Females (n=308) & Females $(\mathrm{n}=308)$ & Males $(\mathrm{n}=216)$ \\
\hline White & $279(90.6)$ & $198(91.7)$ \\
Asian & $9(2.9)$ & $4(1.9)$ \\
Hispanic & $13(4.2)$ & $8(3.7)$ \\
Black & $7(2.3)$ & $6(2.8)$ \\
Previous children, median (range) & $0(0-3)$ & $0(0-2)$ \\
Highest education n (\%) & & \\
Postsecondary and tertiary & $124(40.3)$ & $81(37.5)$ \\
Upper secondary & $146(47.4)$ & $112(51.9)$ \\
Lower secondary of less & $38(12.3)$ & $23(10.6)$ \\
Couples (n=187) & Couples $(\mathbf{n}=\mathbf{1 8 7})$ & Couples $(\mathbf{n}=\mathbf{1 8 7})$ \\
Heterologous fertilization n (\%) & $56(29.9 \%)$ & $56(29.9 \%)$ \\
Previous failed IVF cycle, median (range) & $2(0-8)$ & $2(0-8)$ \\
Duration of infertility, mean (\%) & $3.6( \pm 2.1)$ & $3.6( \pm 2.1)$ \\
Etiology of infertility, n (\%) & & \\
Female factor & $81(43.3)$ & $81(43.3)$ \\
Male factor & $62(33.2)$ & $62(33.2)$ \\
Mixed & $26(13.9)$ & $26(13.9)$ \\
Unexplained & $18(9.6)$ & $18(9.6)$ \\
\hline
\end{tabular}

Table 2. Symptom severity of anxiety and depression of the study population

\begin{tabular}{lllll}
\hline Symptom Severity & Symptom Severity & Females & Females & Females \\
\hline & & Number & Absolute \% & Cumulativ \\
& ANXIETY (GAD-7) & ANXIETY (GAD-7) & ANXIETY (GAD-7) & ANXIET \\
Mild & Mild & 26 & 5.0 & 5.0 \\
Moderate & Moderate & 46 & 8.8 & 13.7 \\
Severe & Severe & 3 & 0.6 & 14.4 \\
TOT & TOT & 75 & 14.4 & - \\
& DEPRESSION (PHQ-9) & DEPRESSION (PHQ-9) & DEPRESSION (PHQ-9) & DEPRES \\
Mild & Mild & 19 & 3.6 & 3.6 \\
Moderate & Moderate & 46 & 8.8 & 12.4 \\
Moderately severe & Moderately severe & 0 & 0 & 12.4 \\
Severe & Severe & 0 & 0 & 12.4 \\
\hline
\end{tabular}

GAD-7: Generalized Anxiety Disorder-7; PHQ-9: Patient Health Questionnaire-9.

*Comparison of symptom severity between males and females

Table 3. Significant variables associate with anxiety/depression at binomial logistic regression

\begin{tabular}{llll}
\hline Variable & B & S.E. & Wald \\
\hline Females/males & Females/males & Females/males & Females/mal \\
Time spent on COVID-19 related news per day (>1 hour per day) & 0.548 & 0.259 & 4.496 \\
Partner with evidence of psychological disorder & 0.699 & 0.293 & 5.709 \\
Females & Females & Females & Females \\
Age & 0.523 & 0.259 & 4.090 \\
Poor ovarian reserve & 0.508 & 0.261 & 3.789
\end{tabular}




\begin{tabular}{|c|c|c|c|}
\hline Variable & B & S.E. & Wald \\
\hline Diagnosis of endometriosis & 0.598 & 0.287 & 4.351 \\
\hline Diagnosis of uterine fibroids & 1.116 & 0.293 & 14.478 \\
\hline
\end{tabular}

\section{RESPONDERS}

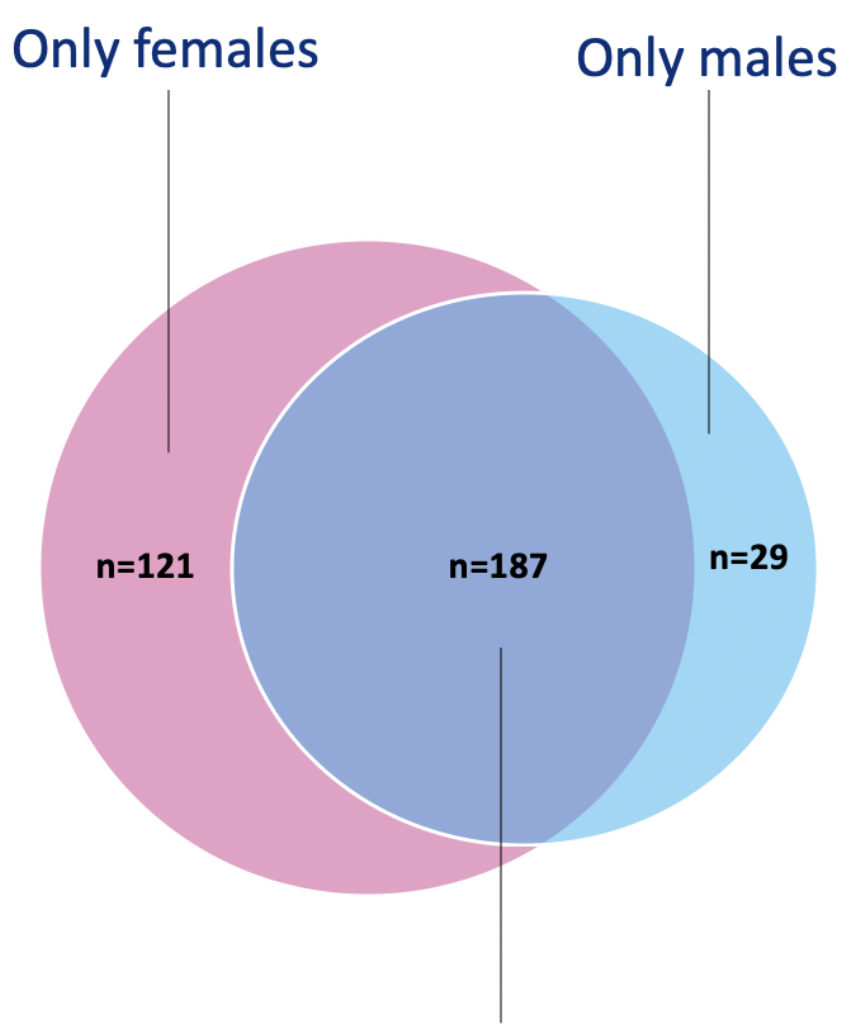

Couples 

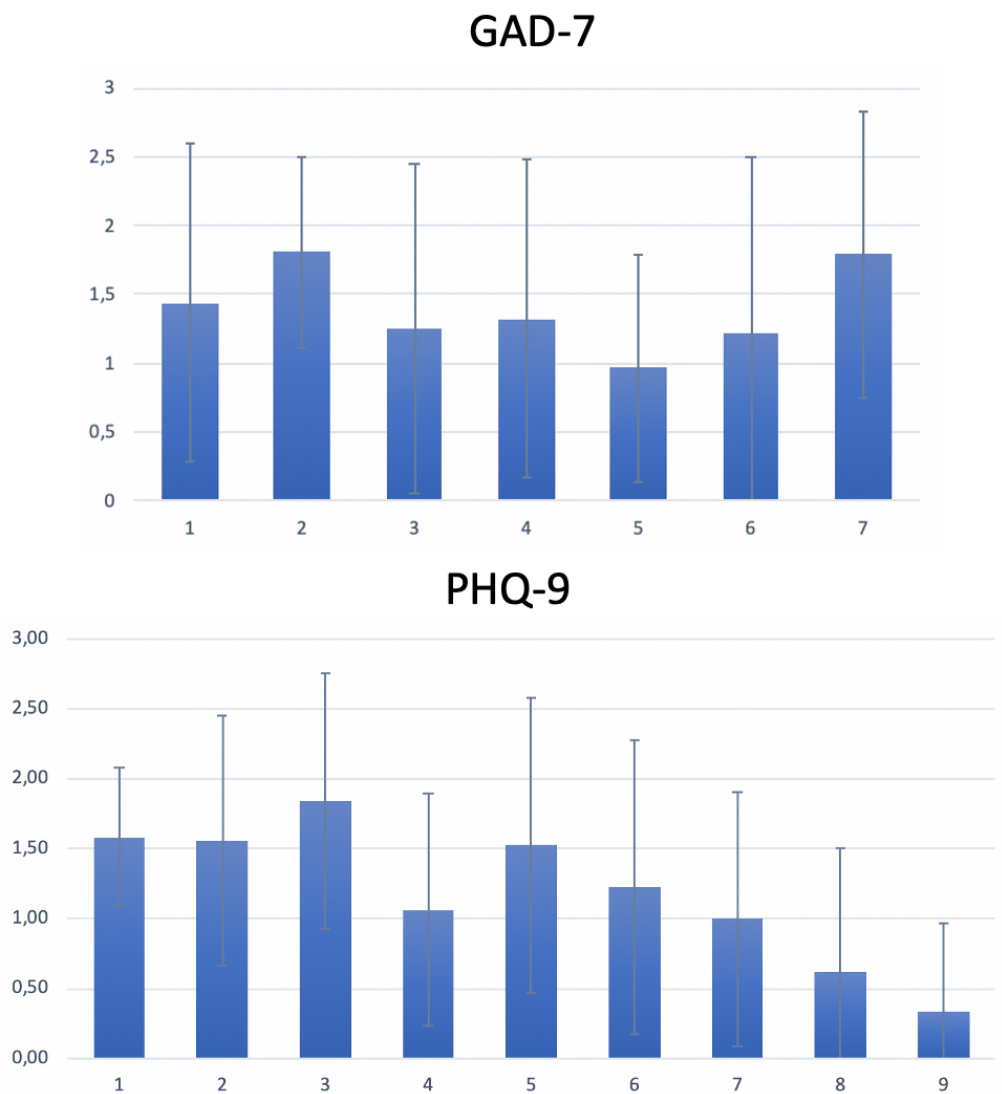